\title{
UNIFORMLY CONTINUOUS FUNCTIONALS ON THE FOURIER ALGEBRA OF ANY LOCALLY COMPACT GROUP
}

\author{
BY
}

\author{
ANTHONY TO-MING LAU ${ }^{1}$
}

\begin{abstract}
Let $G$ be any locally compact group. Let $V N(G)$ be the von Neumann algebra generated by the left regular representation of $G$. We study in this paper the closed subspace $U B C(\hat{G})$ of $V N(G)$ consisting of the uniformly continuous functionals as defined by E. Granirer. When $G$ is abelian, $U B C(\hat{G})$ is precisely the bounded uniformly continuous functions on the dual group $\hat{G}$. We prove among other things that if $G$ is amenable, then the Banach algebra $U B C(\hat{G})^{*}$ (with the Arens product) contains a copy of the Fourier-Stieltjes algebra in its centre. Furthermore, $U B C(\hat{G})^{*}$ is commutative if and only if $G$ is discrete. We characterize $W(\hat{G})$, the weakly almost periodic functionals, as the largest subspace $X$ of $V N(G)$ for which the Arens product makes sense on $X^{*}$ and $X^{*}$ is commutative. We also show that if $G$ is amenable, then for certain subspaces $Y$ of $V N(G)$ which are invariant under the action of the Fourier algebra $A(G)$, the algebra of bounded linear operators on $Y$ commuting with the action of $A(G)$ is isometric and algebra isomorphic to $X^{*}$ for some $X \subseteq U B C(\hat{G})$.
\end{abstract}

1. Introduction. Let $G$ be a locally compact group and let $V N(G)$ denote the von Neumann algebra generated by the left regular representation of $G$, i.e. the closure of the operators $\left\{\lambda(f) ; f \in L_{1}(G)\right\}$ on $L_{2}(G)$ where $\lambda(f)(h)=$ $f * h$ for each $h \in L_{2}(G)$ in $\mathscr{B}\left\{L_{2}(G)\right\}$ the algebra of bounded linear operators from $L_{2}(G)$ into $L_{2}(G)$ in the weak operator topology. Let $A(G)$ denote the linear subspace of $C_{0}(G)$ (bounded continuous complex-valued functions on $G$ vanishing at infinity) consisting of all functions of the form $h * \tilde{k}$ where $h, k \in L_{2}(G)$, and $\tilde{k}(x)=\overline{k\left(x^{-1}\right)}$. Each $\phi=h * \tilde{k}$ in $A(G)$ can be regarded as an ultraweakly continuous linear functional on $V N(G)$ defined by

$$
\phi(T)=\langle T h, k\rangle \text { for each } T \in V N(G) .
$$

P. Eymard [10, p. 210 and p. 218] proved that each ultraweakly continuous linear functional on $V N(G)$ is of this form. Furthermore, $A(G)$ with point-

Received by the editors June 2, 1976 and, in revised form, December 3, 1976.

AMS (MOS) subject classifications (1970). Primary 43A60, 43A30, 43A35, 22D05, 22D15; Secondary 43A07, 22D25, 42A18.

Key words and phrases. Locally compact group, amenable group, regular representation, Fourier algebra, Fourier-Stieltjes algebra, positive definite function, $C^{*}$-group algebra, almost periodic functionals, uniformly continuous functionals, multipliers, invariant mean, second conjugate algebra, Arens product.

${ }^{1}$ This research is supported by NRC Grant A-7679. 
wise multiplication and the norm $\|\phi\|=\sup \{\|\phi(x)\|\}$ where the supremum runs through all $x \in V N(G)$ with $\|x\| \leqslant 1$ is a commutative Banach algebra, called the Fourier algebra of $G$.

There is a natural module action of $A(G)$ on $V N(G)$ given by: $\langle\phi \cdot x$, $\gamma\rangle=\langle\phi \gamma, x\rangle$ for each $\phi, \gamma \in A(G)$ and each $x \in V N(G)$. Then $\|\phi \cdot x\|<$ $\|\phi\|\|x\|[10$, p. 224]. Recently E. Granirer [12] has defined the subspace $U B C(\hat{G})$ of $V N(G)$ as the norm closure of $A(G) \cdot V N(G)$. Then $U B C(\hat{G})$ is a linear space (see footnote on p. 373 in [12]) and elements in $U B C(\hat{G})$ are called uniformly continuous functionals on $A(G)$. In case $G$ is abelian then $U B C(\hat{G})$ is isometric algebra isomorphic to the algebra of bounded uniformly continuous function on the dual group $\hat{G}$ of $G$. Granirer [12, Proposition 1] showed that if $G$ is amenable then $U B C(\hat{G})=A(G) \cdot V N(G)$. In this case, $U B C(\hat{G})$ contains (as in the abelian case) $W(\hat{G})$ the weakly continuous functionals on $A(G)$ as defined by Dunkl and Ramirez [7]. Furthermore, the quotient space $U B C(\hat{G}) / W(\hat{G})$ is not norm separable unless $G$ is discrete. If $G$ is amenable and discrete then $U B C(\hat{G})=W(\hat{G})=A P(\hat{G})$ where $A P(\hat{G})$ is the almost periodic functionals on $G$ (see §2). Figà-Talamanca has proved that if $G$ is the free group on two generators, then $A(G) \cdot V N(G)$ is not closed, and hence not equal to $U B C(G)$. (We would like to thank Professor E. Granirer for kindly informing us of this result, and Professor Figà-Talamanca for communicating the proof to us.)

It is the purpose of this paper to extend the study of the space $U B C(\hat{G})$ and other related subspaces of $V N(G)$. In $\S 3$, we shall discuss various notions of convergence on $B(G)$ that are important to us. In $\S 4$, we shall outline in greater detail the relationship among $U B C(\hat{G})$ and other subspaces of $V N(G)$. We show in particular that (in case $G$ is amenable) $U B C(\hat{G})$ is isometric isomorphic to a closed subspace of $B(G)^{*}$, where $B(G)$ denotes the linear span of the continuous positive definite functions on $G$. In $\$ 5$, we define on each of the dual spaces $U B C(\hat{G})^{*}, W(\hat{G})^{*}$ and $A P(\hat{G})^{*}$ the Arens product turning them into a Banach algebra. We show that $U B C(\hat{G})^{*}$ is commutative if and only if $G$ is discrete. We also show that $W(\hat{G})$ (resp. $A P(\hat{G}))$ is the maximal subspace $X$ of $V N(G)$ for which the Arens product makes sense on $X^{*}$ and the product on $X^{*}$ is separately (resp. jointly) continuous with respect to the weak ${ }^{*}$-topology on bounded spheres of $X^{*} . \$ 6$ is devoted to the study of operators on certain invariant subspaces $X$ of $V N(G)$ commuting with the action of $A(G)$ on $X$ (i.e. $T(\phi \cdot x)=\phi \cdot T(x)$ for each $\phi \in A(G), x \in X)$ with $G$ amenable. We show that the algebra of such operators on $X$ can be realised as the dual space of certain subspaces of $U B C(\hat{G})$. In particular, operators on $V N(G)$ commuting with the action of $A(G)$ is isometric to $U B C(\hat{G})^{*}$. This later result has been obtained independently by Carlo Cecchini (private communication). It generalises a result of 
Curtis and Figà-Talamanca [4, Theorem 3.3] for abelian $G$. We identify those $T$ on $V N(G)$ commuting with the action of $A(G)$ and ultraweakly continuous with $B(G)$, and those $T$ that are in additional compact (or equivalently weakly compact) with $A(G)$ when $G$ is discrete. Finally in $\$ 7$ we show that if $G$ is amenable, then means on $U B C(\hat{G})$ (i.e. elements $\phi \in U B C(\hat{G})$ such that $\|\phi\|=\phi(1)=1$ ) can be approximated by extreme points of the set $P_{1}(G)$, continuous positive definite functions $\phi$ such that $\phi(e)=1$, in the weak*topology of $U B C(\hat{G})^{*}\left(P_{1}(G)\right.$ considered as functionals on $\left.U B C(\hat{G})\right)$. Applications are given to characterize closed convex subsets $A(G)$ invariant under action of $A(G)$ and multipliers on $A(G)$.

It is our pleasure to thank Professor Granirer for his many valuable suggestions on the first version of this paper and also for bringing our attention to the work of $\mathrm{K}$. McKennon.

2. Preliminaries. Let $E$ be a linear space, and $\phi$ be a linear functional on $E$, then the value of $\phi$ at an element $x$ in $E$ will be written as $\phi(x)$ or $\langle\phi, x\rangle$. If $F$ is a subspace of the algebraic dual of $E$, then $\sigma(E, F)$ will denote the weakest locally convex topology on $E$ such that each of the functionals in $F$ is continuous.

If $K$ is a subset of a [normed] linear space $E$, then co $K[\overline{c o} K]$ will denote the [closed convex hull] convex hull of $K$.

Throughout this paper, $G$ denotes a locally compact group with a fixed left Haar measure $\mu$. Integration with respect to $\mu$ will be denoted by $\int \ldots d x$. Let $C(G)$ denote the Banach space of bounded continuous complex-valued functions on $G$ with the supremum norm. Then $G$ is amenable if there exists a positive linear functional $\phi$ on $C(G)$ of norm one such that $\phi\left({ }_{a} f\right)=\phi(f)$ for each $a \in G$ and each $f \in C(G)$, where ${ }_{a} f(t)=f(a t)$ for each $t \in G$. Amenable groups include all solvable groups and all compact groups. However, the free group on two generators is not amenable. For more information on the subject we refer our readers to Geeenleaf's excellent survey [13].

As usual, we denote $C^{*}(G)$ to be the completion of $L_{1}(G)$ with respect to the norm $\|f\|_{c}=\sup \left\{\left\|T_{f}\right\|\right\}$ where the supremum is taken over all nowhere trivial *-representations $T$ of $L_{1}(G)$ as an algebra of bounded operators on Hilbert space. Also, we let $C_{\rho}^{*}(G)$ denote the closure of $\left\{\lambda(g) ; f \in L_{1}(G)\right\}$ in $\mathscr{B}\left(L_{2}(G)\right)$, the algebra of bounded linear operators from $L_{2}(G)$ into $L_{2}(G)$, and $\lambda(f)(h)=f * h$ for each $h \in L_{2}(G)$. Clearly $\|f\|_{c}>\|\lambda(f)\|$ for each $f \in L_{1}(G)$. The two norms agree if and only if $G$ is amenable (see [13, p. 63]).

Let $P(G)$ denote the subspace of $C(G)$ consisting of all continuous positive definite functions on $G$, and let $B(G)$ be its linear span. Then $B(G)$ can be identified with the dual of $C^{*}(G)$, and $P(G)$ is precisely the positive linear functionals on $C^{*}(G)$. Also if $f \in L_{1}(G)$, and $\phi \in B(G)$, then [10, p. 192] $\langle\phi, f\rangle=\int f(t) \phi(t) d t$. 
As proved in [10, Proposition 2.16], $B(G)$ with pointwise multiplication and the dual norm:

$$
\|\phi\|=\sup \left\{\left|\int f(t) \phi(t) d t\right| ; f \in L_{1}(G) \text { and }\|f\|_{c} \leqslant 1\right\}
$$

is a commutative Banach algebra called the Fourier-Stieltjes algebra of $G$.

Let $P_{\rho}(G)$ denote the closure of $P(G) \cap C_{00}(G)$ in the compact open topology, where $C_{00}(G)$ denotes all functions in $C(G)$ with compact support, and let $B_{\rho}(G)$ denote the linear span of $P_{\rho}(G)$. Then $B_{\rho}(G)$ is a closed ideal in $B(G)$ and $B_{\rho}(G)$ is precisely the dual of $C_{\rho}^{*}(G)[10$, p. 191 and Propositions 2.1 and 2.16]. Also, if $\phi \in B_{\rho}(G)$, then the norm of $\phi$ as an element of $B(G)$ is the same as

$$
\sup \left\{\left|\int f(t) \phi(t) d t\right| ; f \in L_{1}(G),\|\lambda(f)\|_{1}<1\right\}
$$

(see [10, p. 193]). As known $[13$, p. 61$], B_{\rho}(G)=B(G)$ if and only if $G$ is amenable.

The Fourier algebra $A(G)$ as defined in $\S 1$ is the closed linear span of $P(G) \cap C_{00}(G)$. Then $A(G) \subseteq B_{\rho}(G)$ and it is a closed ideal in $B(G)$ (see $[10$, p. 208]).

The set of all $x$ in $V N(G)$ for which the operator from $A(G)$ to $V N(G)$ given by $\phi \rightarrow \phi \cdot x$ is weakly compact [compact] is denoted by $W(\hat{G})$ $[A P(\hat{G})]$, the weakly almost periodic [almost periodic] functionals in $V N(G)$. (See Dunkl and Ramirez [7, Definition 2.1, Proposition 2.2 and Chapters 7, 8].)

If $X \subseteq V N(G)$ is a closed subspace of $V N(G)$ containing $1=\lambda(e)$ such that $\phi \cdot X \subseteq X$ for each $\phi \in A(G)$, a linear functional $m$ on $X$ is called a topological invariant mean if $\|m\|=m(1)=1$ and $m(\phi \cdot x)=m(x)$ for each $\phi \in P(G) \cap A(G)$ and $\phi(e)=1$. It has been shown by Renaud [25] that $V N(G)$ always has a topological invariant mean. Furthermore, the topological invariant mean is unique if and only if $G$ is discrete. Also Dunkl and Ramirez [7, Theorem 2.11 and Chapter 8] proved that the subspace $W(\hat{G})$ always has a unique topological invariant mean (see also [12, Proposition 5]).

3. Convergence in $B(G)$. In this section, we discuss various notions of convergence in $B(G)$. Following [3], we let $w^{\sim}$ denote the topology on $B(G)$ such that convergence of a net $\left\{\phi_{\alpha}\right\}$ in $B(G)$ to an element $\phi$ in $B(G)$ means

(i) $\left\langle\phi_{\alpha}, x\right\rangle$ converges to $\langle\phi, x\rangle$ for each $x \in C^{*}(G)$,

(ii) $\left\|\phi_{\alpha}\right\|$ converges to $\|\phi\|$.

LEMMA 3.1. Let $K$ be a compact subset of $G$ and let $\left\{\phi_{\alpha}\right\}$ be a net of states in $A(G)$ with support in $K$. If $\left\{\phi_{\alpha}\right\}$ converges uniformly to some $\phi$ in $A(G)$, then $\phi_{\alpha}$ converges to $\phi$ in norm. 
Proof. Let $\left\{h_{\beta}\right\} \subseteq C_{00}(G)$ be an approximate identity in $L_{1}(G)$ such that $\left\|h_{\beta}\right\|_{1} \leqslant 1$. By passing to a subnet if necessary, we may assume $z_{\beta}$, the image of $h_{\beta}$ in $V N(G)$, converges to $1=\lambda(e)$ in the ultraweak topology. Let $\varepsilon>0$. Then since

$$
\lim _{\beta} \phi\left(\left(1-z_{\beta}\right)^{*}\left(1-z_{\beta}\right)\right)=\lim _{\beta} \phi\left(1-z_{\beta}^{*} z_{\beta}\right)=0
$$

(note that $\left|\phi\left(z_{\beta}\right)\right|^{2} \leqslant \phi\left(\mathrm{z}_{\beta}^{*} z_{\beta}\right) \leqslant 1$ and $\phi\left(z_{\beta}\right) \rightarrow \phi(1)=1$ ), we may find $\beta_{0}$ such that $\phi\left(\left(1-z_{\beta_{0}}\right)^{*}\left(1-z_{\beta_{0}}\right)\right)^{1 / 2}<\varepsilon / 4$. Now for each $\alpha$ and each $x \in V N(G)$ with $\|x\| \leqslant 1$, we have

$$
\begin{aligned}
&\left|\left\langle\phi_{\alpha}-\phi, x-x \cdot z_{\beta_{0}}\right\rangle\right| \leqslant\left|\left\langle\phi_{\alpha}, x\left(1-z_{\beta_{0}}\right)\right\rangle\right|+\left|\left\langle\phi, x\left(1-z_{\beta_{0}}\right)\right\rangle\right| \\
&\left\langle\phi_{\alpha}\left(\left(1-z_{\beta_{0}}\right)^{*}\left(1-z_{\beta_{0}}\right)\right)^{1 / 2}+\phi\left(\left(1-z_{\beta_{0}}\right)^{*}\left(1-z_{\beta_{0}}\right)\right)^{1 / 2}\right.
\end{aligned}
$$

by an application of the Cauchy-Schwarz inequality. Since $\left\langle\phi_{\alpha}-\phi, f\right\rangle \rightarrow 0$ for each $f \in L_{1}(G)$ and $\phi_{\alpha}(1) \rightarrow \phi(1)$, it follows that we may find $\alpha_{0}$ such that if $\alpha>\alpha_{0}$, and $x \in V N(G),\|x\| \leqslant 1$, then

$$
\left|\left\langle\phi_{\alpha}-\phi, x-x \cdot z_{\beta_{0}}\right\rangle\right|<\varepsilon / 2 \text {. }
$$

Let $\alpha_{1}$ be chosen such that $\alpha_{1}>\alpha_{0}$, and if $\alpha>\alpha_{1}$, then $\left|\phi_{\alpha}(t)-\phi(t)\right|<$ $\varepsilon / A$ for all $t \in K$ with $A=2\left(\left\|h_{\beta_{0}}\right\|_{2}+1\right)(m(K)+1)^{1 / 2}$, then for each $f \in$ $L_{1}(G),\|\lambda(f)\| \leqslant 1$ and $\alpha \geqslant \alpha_{1}$, we have

$$
\begin{aligned}
\left|\left\langle\phi_{\alpha}-\phi, f * h_{\beta_{0}}\right\rangle\right| & <\left\|\phi_{\alpha}-\phi\right\|_{2}\left\|f * h_{\beta_{0}}\right\|_{2} \\
& \leqslant\left\|\phi_{\alpha}-\phi\right\|_{2}\left\|h_{\beta_{0}}\right\|_{2}<\varepsilon / 2 .
\end{aligned}
$$

Hence $\left|\left\langle\phi_{\alpha}-\phi, f\right\rangle\right|\left\langle\varepsilon\right.$ for all $f \in L_{1}(G)$ with $\|\lambda(f)\|\langle 1$ and all $\alpha\rangle \alpha_{1}$. An application of the Kaplansky density theorem shows that $\left\|\phi_{\alpha}-\phi\right\|<\varepsilon$ for all $\alpha \geqslant \alpha_{1}$.

THEOREM 3.2. Let $\left\{\phi_{\alpha}\right\}$ be a net in $P(G)$, and $\phi \in P(G)$. The following are equivalent:

(a) $\phi_{\alpha}$ converges to $\phi$ in the compact open topology.

(b) $\phi_{\alpha}$ converges to $\phi$ in the $w^{- \text {-topology. }}$

(c) $\left\|\phi_{\alpha} \psi-\phi \cdot \psi\right\| \rightarrow 0$ for each $\psi \in A(G)$.

(d) $\left\langle\phi_{\alpha} \psi-\phi \psi, x\right\rangle \rightarrow 0$ for each $\psi \in A(G)$ and each $x \in V N(G)$.

PROof. The equivalence of (a) and (b) is precisely Raikov's theorem [24]. That (b) implies (c) is due to McKennon [23, Theorem 5.5]. By first considering elements $\psi$ in $P(G) \cap C_{00}(G)$ it can easily be seen to follow from Lemma 3.3. That (c) implies (d) is trivial.

Finally if (d) holds, and $f \in C_{00}(G)$, choose $\psi \in A(G)$ which assumes the value one on the support of $f$ (see [10, p. 208]). Then $\left\langle\phi_{\alpha} \cdot \psi, f\right\rangle=\left\langle\phi_{\alpha}, f\right\rangle$ converges to $\langle\phi \cdot \psi, f\rangle=\langle\psi, f\rangle$. Also, if $e$ is the identity of $G$, and $\eta$ is a state, then $\left\langle\phi_{\alpha} \eta, 1\right\rangle=\phi_{\alpha}(e)=\left\|\phi_{\alpha}\right\|$ converges to $\langle\phi \cdot \eta, 1\rangle=\phi(e)=\|\phi\|$. Since the 
$\sigma\left(B(G), C_{00}(G)\right)$ agrees with the weak*-topology on bounded spheres, we have $\phi_{\alpha} \rightarrow \phi$ in the $w^{\sim}$-topology; hence (b) holds.

4. $U B C(\hat{G})$ and other subspaces. We denote by $C_{\delta}^{*}(G)$ the $C^{*}$-subalgebra of $\mathscr{B}\left(L_{2}(G)\right)$ generated by the left translations $\{\lambda(g) ; g \in G\}$ on $L_{2}(G)$, where $\lambda(g) f(t)=f(g t)$ for each $t \in G$ and $f \in L_{2}(G)$. We first establish some relationships between $U B C(\hat{G})$ and the $C^{*}$-algebras $C_{\delta}^{*}(G), C_{\rho}^{*}(G)$. We begin with:

Proposition 4.1. (a) The $C^{*}$-algebra $C_{\delta}^{*}(G)$ is contained in $A P(\hat{G})$.

(b) The $C^{*}$-algebra $C_{\rho}^{*}(G)$ is contained in $W(\hat{G})$.

Proof. (a) Let $g \in G$ be fixed. If $\gamma \in A(G)$ and $\|\gamma\|<1$, then for each $\phi \in A(G)$,

$$
\langle\gamma \cdot \lambda(g), \phi\rangle=\langle\lambda(g), \phi \gamma\rangle=\phi(g) \gamma(g)=\langle\gamma(g) \lambda(g), \phi\rangle .
$$

Consequently $\{\gamma \cdot \lambda(g) ;\|\gamma\| \leqslant 1\} \subseteq\{a \cdot \lambda(g) ;|a|<1\}$ which is relatively norm compact and $\lambda(g) \in A P(\hat{G})$. Since $g$ is arbitrary, it follows that $C_{\delta}^{*}(G) \subseteq A P(\hat{G})$.

(b) is due to Dunkl and Ramirez (see [7, Theorem 2.8 and Chapter 8]). For the sake of completeness, we provide a proof.

It suffices to show that if $f \in L_{1}(G)$, then $\lambda(f) \in W(\hat{G})$. Let $f \in L_{1}(G)$ be fixed. If $\phi \in B_{\rho}(G)$, then

$$
\langle\phi \lambda(f), \gamma\rangle=\int f(t) \phi(t) \gamma(t) d t=\langle\lambda(\phi f), \gamma\rangle
$$

for each $\gamma \in A(G)$. Consider the map from $B_{\rho}(G)$ into $V N(G)$ defined by $\phi \rightarrow \lambda(\phi \cdot f)$.

This map is continuous when $B_{\rho}(G)$ has the $\sigma\left(B_{\rho}(G), C_{\rho}^{*}(G)\right)$ topology and $V N(G)$ has the weak topology. In fact, let $\left\{\phi_{\alpha}\right\}$ be a net in $B_{\rho}(G)$ such that $\left\langle\phi_{\alpha}, x\right\rangle \rightarrow\langle\phi, x\rangle$ for each $x \in C_{\rho}^{*}(G)$, then for each functional $l$ in $V N(G)^{*}$, let $\psi \in B_{\rho}(G)$ such that $\langle l, \lambda(h)\rangle=\int h(t) \psi(t) d t$ for each $h \in$ $L_{1}(G)$. Hence

$$
\left\langle\lambda\left(\phi_{\alpha} \cdot f\right), l\right\rangle=\int \phi_{\alpha}(t) f(t) \psi(t) d t=\left\langle\psi \cdot f, \phi_{\alpha}\right\rangle
$$

which converges to $\langle\psi \cdot f, \phi\rangle=\langle\phi \cdot f, l\rangle$. It follows that $\left\{\phi \cdot \lambda(f) ; \phi \in B_{\rho}(G)\right.$ and $\|\phi\|<1\}$ is relatively compact in the weak topology of $V N(G)$. Since $A(G) \subseteq B_{\rho}(G)$, the assertion follows.

Let $W_{0}(\hat{G})$ denote the closed linear subspace of $W(\hat{G})$ consisting of all $x \in W(\hat{G})$ such that $m(x)=0$, where $m$ denotes the unique invariant mean on $W(\hat{G})$. Using Proposition 3.1 and the proof of Theorem 12 in [25] we have:

Proposition 4.2. If $G$ is nondiscrete, then $C_{\rho}^{*}(G) \subseteq W_{0}(\hat{G})$. 
Corollary 4.3. $G$ is discrete if and only if $\lambda(e) \in C_{\rho}^{*}(G)$.

Proof. If $G$ is discrete then $C_{\rho}^{*}(G)=C_{\delta}^{*}(G)$. If $\lambda(e) \in C_{\rho}^{*}(G)$, then $m(\lambda(e))=1$ which implies that $G$ must be discrete by Proposition 4.2.

REMARK. Corollary 4.3 is precisely the Corollary in [3, p. 457] when $G$ is amenable, since in this case $C^{*}(G)=C_{\rho}^{*}(G)$.

If $G$ is amenable, it follows from Proposition 4.1 and Granirer [12, Proposition 1] that $U B C(\hat{G})$ contains $C_{\delta}^{*}(G)$ and $C_{\rho}^{*}(G)$. The next proposition proves slightly more.

Proposition 4.4. For any locally compact group $G, U B C(\hat{G})$ is $a{ }^{*}$-subspace of $V N(G)$ containing $C_{\delta}^{*}(G)$ and $C_{\rho}^{*}(G){ }^{2}$

Proof. To see that $U B C(\hat{G})$ is a *-subspace, it is sufficient to show that if $y=\phi \cdot x$, where $\phi \in A(G)$, and $\phi$ is positive, and $x \in V N(G)$, then $y^{*} \in$ $U B C(\hat{G})$. Indeed, if $\gamma \in A(G)$, and $\gamma$ is positive, then

$$
\left\langle y^{*}, \gamma\right\rangle=\overline{\langle y, \gamma\rangle}=\overline{\langle\phi \cdot x, \gamma\rangle}=\overline{\langle x, \phi \gamma\rangle}=\left\langle\phi \cdot x^{*}, \gamma\right\rangle \text {. }
$$

Hence $y^{*} \in U B C(\hat{G})$.

If $g \in G$, let $\phi \in A(G)$ such that $\phi(e)=1$. Let $\psi(t)=\phi(g t)$ for each $t \in G$, then $\psi \in A(G)[10$, p. 199] and $\psi(g)=1$. Then $\psi \cdot \lambda(g)=\lambda(g)$. Hence $\lambda(g) \in U B C(\hat{G})$. Since $g$ is arbitrary, it follows that $C_{\delta}^{*}(G) \subseteq$ $U B C(\hat{G})$.

Also if $f \in C_{00}(G)$ vanishes outside the compact set $\sigma$, let $\phi \in A(G)$ such that $\phi(t)=1$ for all $t \in \sigma$ (see [10, p. 208]). Then $\phi \cdot \lambda(f)=\lambda(f)$. Hence $\lambda(f) \in U B C(\hat{G})$. Consequently $C_{0}^{*}(G) \subseteq U B C(\hat{G})$.

Proposition 4.5. If $G$ is discrete, then $C_{\rho}^{*}(G)=C_{\delta}^{*}(G)=U B C(\hat{G})$. Conversely, if $U B C(\hat{G})$ is contained in $C_{\rho}^{*}(G)$ or $C_{\delta}^{*}(G)$, then $G$ is discrete.

Proof. Assume that $G$ is discrete, and $a \in G$. Let $1_{a}$ denote the function which is one at $a$ and zero elsewhere. Then $1_{a} \in A(G)[10,3.2$, p. 208]. Let $x \in V N(G)$. Then for each $\phi \in A(G),\left\langle 1_{a} \cdot x, \phi\right\rangle=\left\langle x, \phi(a) 1_{a}\right\rangle=$ $\phi(a)\left\langle x, 1_{a}\right\rangle=\langle c \lambda(a), \phi\rangle$ where $c=\left\langle x, 1_{a}\right\rangle$. Hence $1_{a} \cdot x=c \lambda(a) \in C_{\delta}^{*}(G)$.

Let $\gamma \in A(G)$, and $\gamma_{n} \in A(G)$ be elements with compact support such that $\left\|\gamma_{n}-\gamma\right\| \rightarrow 0$ (see [10, p. 208(3.4)]). Then $\left\|\gamma_{n} \cdot x-\gamma \cdot x\right\| \rightarrow 0$. Since $\gamma_{n} \cdot x \in$ $C_{\delta}^{*}(G)$ for each $n$ by the above argument, it follows that $\gamma \cdot x \in C_{\delta}^{*}(G)$. Consequently $U B C(\hat{G}) \subseteq C_{\delta}^{*}(G)$. By Proposition 4.4, $U B C(\hat{G})=C_{\delta}^{*}(G)=$ $C_{\rho}^{*}(G)$.

Conversely, if $U B C(\hat{G}) \subseteq C_{\delta}^{*}(G)$ or $U B C(\hat{G}) \subseteq C_{\rho}^{*}(G)$, then $U B C(\hat{G})$ is

\footnotetext{
${ }^{2}$ Professor E. E. Granirer has kindly informed us that $U B C(\hat{G})$ is even a $C^{*}$-subalgebra of $V N(G)$ (see Proposition 2(a) in E. E. Granirer, Density theorems for some linear subspaces and some $C^{*}$-subalgebras of $V N(G)$, Symposia Mathematica, Vol. 22, Istituto Nazionale di Alta Matematica, 1977).
} 
contained in $W(\hat{G})$ by Proposition 4.1. Hence by Theorem 12 in Granirer [12], $G$ is discrete.

If $G$ is abelian, then $U B C(\hat{G})$ may be regarded as functionals on $M(\hat{G})$ by defining $\langle\hat{x}, \mu\rangle=\int_{G} x(t) d \mu(t)$ for each $x \in U B C(\hat{G})$ and each regular Borel measure $\mu$ on $\hat{G}$. Since $M(\hat{G})$ includes all point evaluations, it follows that the map $x \rightarrow \hat{x}$ is a linear isometry of $U B C(\hat{G})$ onto a closed subspace of $M(\hat{G})^{*}$. We shall show that for any amenable locally compact group $G$, there exists a linear isometry from $U B C(\hat{G})$ onto a closed subspace of $W^{*}(G)$, the second conjugate algebra of $C^{*}(G)$. Note that $W^{*}(G)$ is isometric isomorphic to $M(\hat{G})^{*}$ when $G$ is abelian.

If $G$ is amenable and $x \in U B C(\hat{G})$ and $x=\phi \cdot y$ with $\phi \in A(G), y \in$ $V N(G)$, define $\hat{x}$ on $B(G)$ by:

$$
\langle\hat{x}, \psi\rangle=\langle y, \phi \psi\rangle \text { for each } \psi \in B(G) .
$$

Then $\hat{x}$ is well defined, since if $x=\gamma \cdot z, \gamma \in A(G)$ and $z \in V N(G)$, then for each $\psi \in A(G)$, we have $\langle y, \phi \psi\rangle=\langle z, \gamma \psi\rangle$. If $\psi \in P(G)$, let $\psi_{\alpha}$ be a net in $P(G) \cap C_{00}(G)$ converging to $\psi$ in the compact open topology, then we have by Theorem 3.2:

$$
\lim _{\alpha}\left\langle y, \phi \psi_{\alpha}\right\rangle=\langle y, \phi \psi\rangle \text { and } \lim _{\alpha}\left\langle z, \gamma \psi_{\alpha}\right\rangle=\langle z, \gamma \psi\rangle \text {. }
$$

Consequently $\langle y, \phi \cdot \psi\rangle=\langle z, \gamma \psi\rangle$. Since the linear span of $P(G)$ is $B(G)$, this last equation holds even for all $\psi \in B(G)$. It is easy to see that $\hat{x}$ is linear on $B(G)$ for each $x$.

THEOREM 4.6. If $G$ is amenable, there exists a linear isometry $\Pi$ from $U B C(\hat{G})$ onto a closed subspace of $W^{*}(G)$ such that $\Pi(x)$ is the point evaluation at a whenever $x=\lambda(a), a \in G$, and $\Pi$ extends the natural embedding of $C^{*}(G)$ into $W^{*}(G)$. Furthermore, $\Pi$ is onto if and only if $G$ is compact.

Proof. If $x \in U B C(\hat{G})$, define $\Pi(x)=\hat{x}$. We first show that $\|x\|=\|\hat{x}\|$. Clearly $\|\hat{x}\| \geqslant\|x\|$. On the other hand, since $G$ is amenable, $A(G)$ has an approximate identity bounded by one (see [21] and Lemma 7.2), given any $\delta>0$, we can find $\phi \in A(G), y \in V N(G)$ such that $x=\phi \cdot y,\|\phi\|<1$ and $\|x-y\| \leqslant \delta$ (see $[15,32.50])$. Hence if $\psi \in B(G)$, and $\|\psi\|<1$, we have:

$$
|\langle\hat{x}, \psi\rangle|=|\langle y, \phi \psi\rangle| \leqslant\|y\| \leqslant\|x\|+\delta .
$$

Consequently, $\|\hat{x}\| \leqslant\|x\|+\delta$. Since $\delta>0$ is arbitrary, it follows that $\|\hat{x}\| \leqslant$ $\|x\|$.

If $a \in G$, and $x=\lambda(a)$, then $x=\phi \cdot x$ where $\phi \in A(G)$ with $\phi(a)=1$. Hence $\langle\hat{x}, \psi\rangle=\langle\phi \cdot x, \psi\rangle=\langle x, \phi \psi\rangle=\psi(a)$.

If $x=\lambda(f)$ and $f \in C_{00}(G)$ vanishing outside the compact set $\sigma$. Let $\phi \in A(G)$ such that $\phi(t)=1$ for each $t \in \sigma$. Then if $\psi \in B(G)$,

$$
\langle\hat{x}, \psi\rangle=\langle x, \phi \psi\rangle=\int f(t) \phi(t) \psi(t) d t=\int f(t) \psi(t) d t=\langle x, \psi\rangle .
$$


Hence $\Pi$ agrees with the natural embedding of $C^{*}(G)$ into $W^{*}(G)$ on the dense set $\left\{\lambda(f) ; f \in C_{00}(G)\right\}$. Consequently $\Pi$ must agree on $C^{*}(G)$.

Now if $G$ is compact, then $1 \in A(G)$ and $x=1 \cdot x$ for each $x \in V N(G)$. Hence $V N(G)=U B C(\hat{G})$ (see [12, p. 373]). Also $A(G)=B(G)$ (see [10, p. 209]). Hence $\Pi(U B C(\hat{G}))=A(G)^{*}=B(G)^{*}=W^{*}(G)$.

Conversely, if $\Pi(U B C(G))=W^{*}(G)$, and $\psi_{0} \in P(G), \psi_{0} \notin A(G)$, let $x=\phi \cdot y, \phi \in A(G), y \in V N(G)$ such that $\left\langle\hat{x}, \psi_{0}\right\rangle \neq 0$, and $\langle\hat{x}, \psi\rangle=0$ for all $\psi \in A(G)$. Let $\psi_{\alpha}$ be a net in $A(G) \cap P(G)$ which converges to $\psi_{0}$ in the

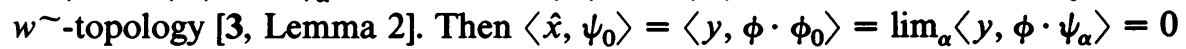
by Theorem 3.2, which is impossible. Hence $P(G) \subseteq A(G)$ and $A(G)=$ $B(G)$. In particular $A(G)$ has an identity. Consequently $G$, which is the spectrum of $A(G)$ [10, Theorem 3.34], must be compact.

5. The Banach algebras $U B C(\hat{G})^{*}, W(\hat{G})^{*}$ and $A P(\hat{G})^{*}$. A subset $X$ of $V N(G)$ is topologically invariant if $\phi \cdot x \in X$ for each $\phi \in A(G)$ and each $x \in X$. If $X$ is a topologically invariant linear subspace of $V N(G)$, we say that $X$ is topologically introverted if for each $m \in X^{*}$ and each $x \in X$, the functional $\gamma \rightarrow\langle m, \gamma \cdot x\rangle$ on $A(G)$, denoted by $m \odot x$, also defines an element in $X$. When $G$ is abelian, our definitions coincide precisely with the topologically invariant and topologically introverted subspaces of $L_{\infty}(\hat{G})$ as defined by Wong [29, p. 356].

LEMMA 5.1. Let $X$ be a topological invariant subspace of $V N(G)$. Then $X$ is topologically introverted if and only if for each $x \in X, \overline{K(x)^{\circ}}$, the ultraweak closure of the set $K(x)=\left\{\phi \cdot x ; \phi \in P_{1}(G) \cap A(G)\right\}$, is contained in $X$, where $P_{1}(G)=\{\phi \in P(G) ;\|\phi\|=1\}$.

Proof. If $X$ is topologically introverted, and $y \in \overline{K(x)}^{\circ}, x \in X$, then there exists a net $\left\{\phi_{\alpha}\right\}$ in $P_{1}(G) \cap A(G)$ such that $\phi_{\alpha} \cdot x$ converges to $y$ in the ultraweak topology. Let $m$ be a weak*-cluster point of $\left\{\phi_{\alpha}\right\}$ in $V N(G)^{*}$. Then for each $\gamma \in A(G)$,

$$
\langle y, \gamma\rangle=\lim _{\alpha}\left\langle\phi_{\alpha} \cdot x, \gamma\right\rangle=\lim _{\alpha}\left\langle\gamma x, \phi_{\alpha}\right\rangle=\langle\gamma x, m\rangle=\langle m \odot x, \gamma\rangle .
$$

Hence $y=m \odot x \in X$. Conversely if $\overline{K(x)}^{\sigma} \subseteq X$ for each $x \in X$, let $m$ be a state on $V N(G)$; we can find a net $\left\{\phi_{\alpha}\right\}$ of ultraweakly continuous states such that $\left\{\phi_{\alpha}\right\}$ converges to $m$ in the weak*-topology of $V N(G)^{*}$ (see [12, Proposition 3]). Consequently if $\gamma \in A(G)$,

$$
\langle m \odot x, \gamma\rangle=\langle m, \gamma \cdot x\rangle=\lim _{\alpha}\left\langle\phi_{\alpha}, \gamma \cdot x\right\rangle=\lim _{\alpha}\left\langle\phi_{\alpha} \cdot x, \gamma\right\rangle \text {. }
$$

Hence $m \odot x \in \overline{K(x)}^{\sigma} \subseteq X$. Since every functional in $X^{*}$ is extendable to a functional on $V N(G)$, and each element in $V N(G)^{*}$ is the linear combination of states, it follows that $m \odot x \in X$ for each $m \in X^{*}$ and each $x \in X$. 
Proposition 5.2. The spaces $U B C(\hat{G}), W(\hat{G}), A P(\hat{G})$ and $C_{p}^{*}(G)$ are topologically invariant and topologically introverted.

Proof. That the spaces are topologically invariant is trivial. To see that $U B C(\hat{G})$ is topologically introverted, let $m \in U B C(\hat{G})^{*}$. If $x=\gamma_{0} \cdot z, \gamma_{0} \in$ $A(G)$ and $z \in V N(G)$, then $m \odot x=\gamma_{0} \cdot(m \odot z)$. Hence $m \odot x \in U B C(\hat{G})$. If $x \in U B C(\hat{G})$, let $\left\{x_{n}\right\}$ be a sequence in $A(G) \cdot V N(G)$ such that $\left\|x_{n}-x\right\|$ $\rightarrow 0$. Then $\left\|m \odot x_{n}-m \odot x\right\| \leqslant\|m\|\left\|x_{n}-x\right\| \rightarrow 0$. Hence $m \odot x \in$ $\operatorname{UBC}(\hat{G})$.

To see that $W(\hat{G}), A P(\hat{G})$ and $C_{\rho}^{*}(G)$ are introverted, it is sufficient, by Proposition 4.1, to show that any closed topologically invariant subspace $X$ of $W(G)$ is topologically introverted. Indeed, let $x \in X$. Using the notation of Lemma 5.1, the convex set $K(x)$ is relatively compact in the weak topology of $V N(G)$. Hence the ultraweak and the weak topology agrees on $\overline{K(x)}$, the norm closure of $K(x)$. Consequently $\overline{K(x)}^{\sigma}=\overline{K(x)}$ is contained in $X$. By Lemma 5.1, $X$ is topologically introverted.

In [1] Arens shows that given a Banach algebra $B$, it is possible to define a multiplication on $B^{* *}$ which extends multiplication on $B$. In case $B=A(G)$, $m, n \in V N(G)^{*}$, the Arens product $m \odot n$ is defined by the formula:

$$
\langle m \odot n, x\rangle=\langle m, n \odot x\rangle \text { for each } x \in V N(G) \text {. }
$$

This same formula certainly makes sense when $V N(G)$ is replaced by a topologically invariant and introverted subspace $X$ of $V N(G)$. This product also turns $X^{*}$ into a Banach algebra. Note that if $m, n \in X^{*}$, and $\tilde{m}, \tilde{n}$ are extensions of $m, n$ to $V N(G)$, then $\tilde{m} \odot \tilde{n}$ is also an extension of $m \odot n$ to $V N(G)$.

Proposition 5.3. Arens' product on $C_{\rho}^{*}(G)^{*}=B_{\rho}(G)$ is precisely the pointwise multiplication on $B_{\rho}(G)$.

Proof. If $\psi \in A(G)$ and $x \in C_{\rho}^{*}(G)$, then $\psi \odot x=\psi \cdot x$. If $\psi \in P_{\rho}(G)$, let $\psi_{\alpha}$ be a net in $P(G) \cap C_{00}(G)$ such that $\psi_{\alpha}$ converges to $\psi$ in the compact open topology. Then by Theorem 3.2, we have:

$$
\begin{aligned}
\langle\psi \odot x, \gamma\rangle & =\langle\psi, \gamma \cdot x\rangle=\lim _{\alpha}\left\langle\psi_{\alpha}, \gamma \cdot x\right\rangle=\lim _{\alpha}\left\langle\psi_{\alpha} \cdot \gamma, x\right\rangle \\
& =\langle\psi \cdot \gamma, x\rangle=\langle\psi \cdot x, \gamma\rangle
\end{aligned}
$$

for each $x \in C_{\rho}^{*}(G)$ and $\gamma \in A(G)$. Since the span of $P_{\rho}(G)$ is $B_{\rho}(G)$, it follows that $\psi \odot x=\psi \cdot x$ for each $\psi \in B_{\rho}(G)$ and each $x \in C_{\rho}^{*}(G)$. Now if $\theta, \psi \in B_{\rho}(G)$ and $x \in C_{\rho}^{*}(G)$, we have

$$
\langle\theta \odot \psi, x\rangle=\langle\theta, \psi \odot x\rangle=\langle\theta, \psi \cdot x\rangle=\langle\theta \cdot \psi, x\rangle .
$$

Hence $\theta \odot \psi=\theta \cdot \psi$.

If $G$ is amenable and $\phi \in B(G)$, let $\hat{\phi}$ denote the functional on $U B C(\hat{G})$ defined by $\langle\hat{\phi}, x\rangle=\langle\Pi x, \phi\rangle$ for each $x \in U B C(\hat{G})$, and $\Pi$ is the linear 
isometry of $U B C(\hat{G})$ into $W^{*}(G)$ as defined in Theorem 4.6. Our next proposition lists some properties of the Arens product $U B C(G)^{*}$ :

Proposition 5.4. (a) For each $m \in U B C(\hat{G})^{*}$, the map $n \rightarrow n \odot m$ from $U B C(\hat{G})^{*}$ into $U B C(\hat{G})^{*}$ is weak*-weak ${ }^{*}$ continuous.

(b) If $G$ is amenable, then for each $\phi \in B(G)$, the map $m \rightarrow \phi \odot m$ from $U B C(\hat{G})^{*}$ into $U B C(\hat{G})^{*}$ is weak ${ }^{*}$-weak ${ }^{*}$ continuous.

(c) If $\phi, \gamma \in B(G)$ and $G$ is amenable, then $\hat{\phi} \gamma=\hat{\phi} \odot \hat{\gamma}$.

Proof. (a) is trivial.

(b) We first observe that if $m \in U B C(\hat{G})^{*}$, then $\langle\hat{\phi} \odot m, x\rangle=\langle m, \phi \cdot x\rangle$ for each $x \in U B C(\hat{G})$. Indeed, if $x$ is of the form $\gamma_{0} \cdot z$ with $\gamma_{0} \in A(G)$ and $z \in V N(G)$, then $m \odot x=\gamma_{0} \cdot(m \odot z)$. Hence

$$
\begin{aligned}
\langle\hat{\phi} \odot m, x\rangle & =\left\langle\hat{\phi}, \gamma_{0} \cdot(m \odot z)\right\rangle \\
& =\left\langle m \odot z, \gamma_{0} \phi\right\rangle=\left\langle m,\left(\gamma_{0} \phi\right) \cdot z\right\rangle=\langle m, \phi \cdot x\rangle .
\end{aligned}
$$

Now let $\left\{m_{\alpha}\right\}$ be a net in $U B C(\hat{G})^{*}$ converging to $m \in U B C(\hat{G})^{*}$ in the weak $^{*}$-topology. Then $\langle\hat{\phi} \odot m, x\rangle=\left\langle m_{\alpha}, \phi \cdot x\right\rangle$ which converges to $\langle m, \phi \cdot$ $x\rangle$ for each $x \in U B C(\hat{G})$, since the element $\phi \cdot x$ is also in $U B C(\hat{G})$.

(c) If $\phi, \gamma \in B(G)$, and $x=\gamma_{0} \cdot z, \gamma_{0} \in A(G)$ and $z \in V N(G)$. Then

$$
\begin{aligned}
\langle\hat{\phi} \odot \hat{\gamma}, x\rangle & =\langle\hat{\phi}, \hat{\gamma} \odot x\rangle=\left\langle\hat{\phi}, \gamma_{0} \cdot(\hat{\gamma} \odot z)\right\rangle=\left\langle\phi \gamma_{0}, \hat{\gamma} \odot z\right\rangle \\
& =\left\langle\phi \gamma_{0} \gamma, z\right\rangle=\langle\hat{x}, \phi \gamma\rangle=\langle\hat{\phi} \gamma, x\rangle .
\end{aligned}
$$

The assertion now follows.

THEOREM 5.5. Assume that $G$ is amenable. Then the map $Q: \phi \rightarrow \hat{\phi}$ is a linear isometry and an algebra homomorphism from $B(G)$ into $U B C(\hat{G})^{*}$. The image of $B(G)$ under $Q$ is contained in the centre of the algebra $U B C(\hat{G})^{*}$. Furthermore, $U B C(\hat{G})^{*}$ is commutative if and only if $G$ is discrete.

Proof. That $Q$ is an algebra homomorphism follows from Proposition 5.4(c). Now if $\phi \in B(G)$, and $x \in U B C(\hat{G})$ with $\|x\|<1$, then

$$
|\langle\hat{\phi}, x\rangle|=|\langle\Pi x, \phi\rangle|<\|\Pi x\|\|\phi\|=\|x\|\|\phi\|<\|\phi\|
$$

by Theorem 4.6. Hence $\|\hat{\phi}\|<\|\phi\|$. On the other hand, since $C^{*}(G) \subseteq$ $U B C(\hat{G})$, and $\langle\hat{\phi}, x\rangle=\langle\phi, x\rangle$ if $x \in C^{*}(G)$ (Theorem 4.6), it follows that $\|\hat{\phi}\|=\|\boldsymbol{\phi}\|$.

If $m \in U B C(\hat{G})^{*}$, let $\left\{\phi_{\alpha}\right\}$ be a net in $A(G)$ such that $\hat{\phi}_{\alpha}$ converges to $m$ in the weak*-topology. Then if $\gamma \in B(G)$, and $x \in U B C(\hat{G})$, we have

$$
\begin{aligned}
\langle m \odot \hat{\gamma}, x\rangle & =\lim _{\alpha}\left\langle\hat{\phi}_{\alpha} \odot \gamma, x\right\rangle=\lim _{\alpha}\left\langle\hat{\phi}_{\alpha} \gamma, x\right\rangle \\
& =\lim _{\alpha}\left\langle\hat{\gamma} \odot \hat{\phi}_{\alpha}, x\right\rangle=\langle\hat{\gamma} \odot m, x\rangle
\end{aligned}
$$

by Proposition 5.4. Hence $\hat{\gamma}$ is contained in the centre of $U B C(\hat{G})^{*}$. 
If $G$ is discrete, then $U B C(\hat{G})=C^{*}(G)$ by Proposition 4.5 . Hence $Q$ is onto and $U B C(\hat{G})^{*}$ is commutative. Conversely, if $U B C(\hat{G})^{*}$ is commutative, let $m_{1}, m_{2}$ be distinct topological invariant means on $V N(G)$; then $m_{1}$, $m_{2}$ are also distinct topological invariant means on $U B C(\hat{G})$. But $m_{1} \odot m_{2}=$ $m_{2}$ and $m_{2} \odot m_{1}=m_{1}$. Hence $m_{2}=m_{1}$ when restricted to $U B C(\hat{G})$, which is impossible. By [25, Theorem 11], $G$ is discrete.

THEOREM 5.6. Let $X$ be a closed topologically invariant and topologically introverted subspace of $V N(G)$. The following are equivalent:

(a) $X \subseteq W(\hat{G})$.

(b) The product in $X^{*}$ is separately continuous with respect to the weak*topology on bounded spheres.

(c) $X^{*}$ is a commutative Banach algebra.

Proof. (a) $\Rightarrow$ (b). It is clear that if $m \in X^{*}$, the map $n \rightarrow n \odot m$ from $X^{*}$ into $X^{*}$ is weak ${ }^{*}$-weak ${ }^{*}$ continuous. To prove continuity in the other variable, let $\left\{n_{\alpha}\right\}$ be a net in $X^{*}$ converging to some $n \in X^{*}$ in the weak*-topology and $\left\|n_{\alpha}\right\| \leqslant M$ and $\|n\| \leqslant M$ for some $M>0$. We may assume that $M=1$. For each $x \in X$, the net $\left\{n_{\alpha} \odot x\right\}$ converges to $n \odot x$ in the ultraweak topology (denoted by $\sigma$ ) on $V N(G)$. Let $K(x)=\{\phi \cdot x ; \phi \in A(G)$ and $\|\phi\| \leqslant 1\}$, and $\overline{K(x)}, \overline{K(x)^{\sigma}}$ denote the norm and $\sigma$-closure of $K(x)$ in $V N(G)$. Since $K(x)$ is relatively compact in the weak topology of $V N(G)$, it follows that the weak and $\sigma$-topologies agree on $\overline{K(x)}$ and $\overline{K(x)}=\overline{K(x)^{\circ}}$. Consequently the net $\left\{n_{\alpha} \odot x\right\}$ (which is in $\overline{K(x)^{\sigma}}$ ) also converges to $n \odot x$ in the weak topology. So if $m \in X^{*}$, then $\left\langle m \odot n_{\alpha}, x\right\rangle=\left\langle m, n_{\alpha} \odot x\right\rangle$ converges to $\langle m, n \odot x\rangle=\langle m \odot n, x\rangle$ also.

(b) $\Rightarrow$ (c). Let $m \in X^{*}, \gamma$ be the restriction of an element in $A(G)$ to $X$, and $\left\{\phi_{\alpha}\right\}$ be a net in $A(G)$ such that $\left\langle\phi_{\alpha}, x\right\rangle$ converges to $\langle m, x\rangle$ for each $x \in X$ and $\left\|\phi_{\alpha}\right\|<\|m\|$ for each $\alpha$, then

$$
\langle m \odot \gamma, x\rangle=\lim _{\alpha}\left\langle\phi_{\alpha} \odot \gamma, x\right\rangle=\lim _{\alpha}\left\langle\gamma \odot \phi_{\alpha}, x\right\rangle=\langle\gamma \odot m, x\rangle
$$

by (b). Hence $m \odot \gamma=\gamma \odot m$. A second application of (b) and the weak*denseness of $A(G)$ (restricted to $X$ ) in $X^{*}$ shows that $m \odot n=n \odot m$ for each $m, n \in X^{*}$.

(c) $\Rightarrow$ (a). Let $x \in X$ and consider the map $p: m \rightarrow m \odot x$ from $X^{*}$ into $X$. If $\left\{m_{\alpha}\right\}$ is a net in $X^{*}$ converging to $m$ in the weak*-topology, then for each $n$ in $X^{*},\left\langle m_{\alpha} \odot x, n\right\rangle=\left\langle n \odot m_{\alpha}, x\right\rangle=\left\langle m_{\alpha} \odot n, x\right\rangle$ converges to $\langle m \odot n, x\rangle=$ $\langle n \odot m, x\rangle=\langle m \odot x, n\rangle$ by (c). Hence $p$ is weak*-weak continuous. Consequently, the set $\left\{m \odot x ; m \in X^{*}\right.$ and $\left.\|m\| \leqslant 1\right\}$, which includes $\{\phi \odot x ; \phi \in A(G)$ and $\|\phi\| \leqslant 1\}$ is relatively compact in the weak topology of $X$ (hence of $V N(G)$ ). So $x \in W(\hat{G})$.

COROLLARY $5.7[7]$. $W(\hat{G})$ has a unique topological invariant mean. 
PROof. If $m_{1}, m_{2}$ are topological invariant means on $W(\hat{G})$, then $m_{2}=$ $m_{1} \odot m_{2}=m_{2} \odot m_{1}=m_{1}$ by Theorem 5.6.

REMARK. See [12, Proposition 5] for a different proof of Corollary 5.7.

The following is an analogue of Theorem 5.6:

THEOREM 5.8. Let $X$ be a closed topologically invariant and topologically introverted subspace of $V N(G)$. The following are equivalent:

(a) $X \subseteq A P(\hat{G})$.

(b) Multiplication in $X^{*}$ is jointly continuous with respect to the weak*topology on bounded spheres.

Proof. (a) $\Rightarrow$ (b). Let $\left\{m_{\alpha}\right\},\left\{n_{\beta}\right\}$ be nets in $X^{*}$ converging to $m, n$ in $X^{*}$ respectively in the weak*-topology such that $\left\|m_{\alpha}\right\|<M$ and $\left\|n_{\beta}\right\|<M$. We may assume that $M=1$. Let $x \in X$. The set $K(x)=\{\phi \cdot x ; \phi \in A(G)$ and $\|\phi\| \leqslant 1\}$ is relatively compact in the norm topology of $V N(G)$. Hence the norm and the ultraweak topologies agree on $\overline{K(x)}$ and $\overline{K(x)}=\overline{K(x)}{ }^{\circ}$. As in the proof of Theorem 5.6, the net $n_{\beta} \odot x$ converges to $n \odot x$ in the norm topology. Hence

$$
\begin{aligned}
\left|\left\langle m_{\alpha} \odot n_{\beta}, x\right\rangle-\langle m \odot n, x\rangle\right| \\
\\
\left\langle\left|\left\langle m_{\alpha} \odot n_{\beta}, x\right\rangle-\left\langle m_{\alpha} \odot n, x\right\rangle\right|+\left|\left\langle m_{\alpha} \odot n, x\right\rangle-\langle m \odot n, x\rangle\right|\right. \\
\quad \leqslant\left\|n_{\beta} \odot x-n \odot x\right\|+\left|\left\langle m_{\alpha}, n \odot x\right\rangle-\langle m, n \odot x\rangle\right|
\end{aligned}
$$

which converges to zero.

(b) $\Rightarrow$ (a). If (b) holds, then $X \subseteq W(\hat{G})$ by Theorem 5.6. Hence if $x \in X$, $\overline{K(x)}=\overline{K(x)}^{\circ}=\left\{m \odot x ; m \in X^{*}\right.$ and $\left.\|m\|<1\right\}$. Let $\left\{m_{\alpha} \odot x\right\}$ be a net in $\overline{K(x)}$. There exists a subnet $\left\{m_{\gamma}\right\}$ of the net $\left\{m_{\alpha}\right\}$ which converges to some $m$ in the weak*-topology. We shall show that the net $\left\{m_{\gamma} \odot x\right\}$ converges to $m \odot x$ in norm. If not, we may by passing to a subnet if necessary assume that there exist $\varepsilon>0$ such that $\left\|m_{\gamma} \odot x-m \odot x\right\|>\varepsilon>0$ for all $\gamma$. For each $\gamma$ pick $n_{\gamma} \in X^{*}$ such that $\left\|n_{\gamma}\right\| \leqslant 1$ and $\left\|m_{\gamma} \odot x-m \odot x\right\|=\mid\left\langle m_{\gamma} \odot x-\right.$ $\left.m \odot x, n_{\gamma}\right\rangle \mid$. Again, by passing to a subnet if necessary, we may assume that the net $\left\{n_{\gamma}\right\}$ converges to some $n \in X^{*}$ in the weak ${ }^{*}$-topology. Now for each $\gamma$,

$$
\begin{aligned}
\varepsilon \leqslant & \left|\left\langle m_{\gamma} \odot x-m \odot x, n\right\rangle\right|\left\langle\left|\left\langle n_{\gamma} \odot m_{\gamma}, x\right\rangle-\langle n \odot m, x\rangle\right|\right. \\
& +\left|\langle n \odot m, x\rangle-\left\langle n_{\gamma} \odot m, x\right\rangle\right|<\varepsilon
\end{aligned}
$$

for $\gamma$ far out enough. But this is impossible. Hence $\left\{m_{\gamma} \odot x\right\}$ converges to $m \odot x$ in norm. Consequently $\overline{K(x)}$ is norm compact, i.e. $x \in A P(\hat{G})$.

6. Operators commuting with action by $A(G)$ on subspaces of $V N(G)$. Let $X$ be a closed topologically invariant subspace of $V N(G)$. Say that an operator $T: X \rightarrow X$ commutes with action by $A(G)$ if it satisfies 


$$
T(\phi \cdot x)=\phi \cdot T(x) \text { for all } \phi \in A(G) \text { and all } x \in X .
$$

We shall now give a characterization of the space of all such operators in terms of the dual of certain subspaces of $U B C(\hat{G})$.

Assume that $G$ is amenable. Then the existence of an approximate identity in $A(G)$ and the Cohen factorization theorem show that $A(G) \cdot X$ is a closed linear subspace of $V N(G)$. Furthermore, $A(G) \cdot X$ is topologically introverted if $X$ is topologically introverted.

For each $m \in(A(G) \cdot X)^{*}$, define a bounded linear operator $m_{L}$ from $X$ into $X$ by $\left\langle m_{L}(x), \gamma\right\rangle=\langle m, \gamma \cdot x\rangle$ for each $\gamma \in A(G)$ and $x \in X$.

LEMMA 6.1. Assume that $G$ is amenable, then:

(a) $m_{L}$ commutes with action of $A(G)$ on $X$.

(b) $\left\|m_{L}\right\|=\|m\|$.

Proof. (a) is trivial.

(b) It is clear that $\left\|m_{L}\right\|<\|m\|$. To prove the converse, let $\left\{\phi_{\alpha}\right\}$ be an approximate identity in $A(G)$ and $\left\|\phi_{\alpha}\right\|<1$. Then for each $z \in A(G) \cdot X$, $\left\|\phi_{\alpha} \cdot z-z\right\| \rightarrow 0$. Hence

$$
\left\|m_{L}(z)\right\|>\left|\left\langle m_{L}(z), \phi_{\alpha}\right\rangle\right|=\left|\left\langle m, \phi_{\alpha} \cdot z\right\rangle\right|
$$

which converges to $|\langle m, z\rangle|$. Hence $\left.\left\|m_{L}\right\|\right\rangle\|m\|$.

TheOREM 6.2. Assume that $G$ is amenable. Let $X$ be a topological invariant and topologically introverted closed subspace of $V N(G)$. Then the map $\tau$ : $m \rightarrow m_{L}$ is a linear isometry and algebra homomorphism from $(A(G) \cdot X)^{*}$ onto the space of all bounded linear operators commuting with the action of $A(G)$ on $X$.

Proof. By Lemma 6.1, it is sufficient to show that $\tau$ is onto. Let $\left\{\phi_{\alpha}\right\}$ be a bounded approximate identity on $A(G)$, and $T$ be a bounded linear operator commuting with action of $A(G)$ on $X$. Then $T$ maps $A(G) \cdot X$ into $A(G) \cdot X$. Let $m$ be a weak*-cluster point of the net $\left\{T^{*}\left(\phi_{\alpha}\right)\right\}$ in $(A(G) \cdot X)^{*}$. Then if $x \in X$, and $\gamma \in A(G)$, we have

$$
\begin{aligned}
\langle T(x), \gamma\rangle & =\lim _{\alpha}\left\langle T(x), \phi_{\alpha} \cdot x\right\rangle=\lim _{\alpha}\left\langle\gamma \cdot T(x), \phi_{\alpha}\right\rangle \\
& =\lim _{\alpha}\left\langle T(\gamma \cdot x), \phi_{\alpha}\right\rangle=\left\langle m_{L}(x), \gamma\right\rangle .
\end{aligned}
$$

Hence $T=m_{L}$. To see that $\tau$ is an algebra homomorphism, let $n, m \in(A(G)$ $\cdot X)^{*}$, then for each $\gamma \in A(G)$ and $x \in X$ :

$$
\begin{aligned}
\left\langle(n \odot m)_{L}(x), \gamma\right\rangle & =\langle(n \odot m), \gamma \cdot x\rangle=\left\langle n, m_{L}(\gamma \cdot x)\right\rangle \\
& =\left\langle n, \gamma \cdot m_{L}(x)\right\rangle=\left\langle n_{L}\left(m_{L}\right)(x), \gamma\right\rangle
\end{aligned}
$$

i.e. $(n \odot m)_{L}=n_{L}\left(m_{L}\right)$. 
COROLlaRY 6.3. If $G$ is amenable, and $X$ is a topologically invariant topologically introverted closed subspace of $U B C(\hat{G})$, then $X^{*}$ is isometric and algebra isomorphic to the algebra of bounded linear operators commuting with action of $A(G)$ on $X$.

Proof. It is sufficient to show that $A(G) \cdot X=X$. Indeed, if $x \in X$, and $\left\{\phi_{\alpha}\right\}$ is an approximate identity for $A(G)$, then $\left\|\phi_{\alpha} \cdot x-x\right\| \rightarrow 0$. Since $A(G) \cdot X$ is closed, $x \in A(G) \cdot X$.

COROLlaRY 6.4. If $G$ is amenable, then $U B C(\hat{G})^{*}$ is isometric and algebra isomorphic with all bounded operators commuting with action of $A(G)$ on $V N(G)$.

COROllary 6.5. If $G$ is amenable, then $B(G)$ is isometric and algebra isomorphic to all bounded operators commuting with action of $A(G)$ on $C^{*}(G)$.

REMARK. Carlo Cecchini has proved independently most of Corollary 6.4: If $G$ is amenable, then $U B C(\hat{G})^{*}$ is isometric with all bounded operators $T$ : $V N(G) \rightarrow V N(G)$ commuting with the action of $A(G)$ (private communication). When $G$ is abelian, Corollary 6.4 is due to Curtis and FigàTalamanca [4]. Also, Theorem 6.2 is an analogue of our result in [19] for subspaces of $L_{\infty}(\hat{G})$.

THEOREM 6.6. If $G$ is amenable, then the map $m \rightarrow \hat{m}_{L}$ is a linear isometry and an algebra isomorphism from $B(G)$ onto the algebra of all ultraweakly continuous bounded linear operators commuting with the action of $A(G)$ on $V N(G)$.

Proof. It is easy to see that if $m \in B(G)$, then $\hat{m}_{L}$ is ultraweakly continuous. Conversely, if $T: V N(G) \rightarrow V N(G)$ is ultraweakly continuous commuting with action of $A(G)$ on $V N(G)$ and $\left\{\phi_{\alpha}\right\}$ is a bounded approximate identity of $A(G)$, then $T^{*}\left(\phi_{\alpha}\right) \in A(G)$ for each $\alpha$. By passing to a subnet if necessary, we may assume that the net $\left\{T^{*}\left(\phi_{\alpha}\right)\right\}$ converges to some $m \in B(G)$ in the weak*-topology of $B(G)$. If $x \in C^{*}(G)$, and $\psi \in B(G)$, then

$$
\begin{aligned}
\left\langle\hat{m}_{L}(x), \psi\right\rangle & =\langle\hat{m}, x \psi\rangle=\langle x, m \psi\rangle=\lim _{\alpha}\left\langle x, T^{*}\left(\phi_{\alpha}\right) \psi\right\rangle \\
& =\lim _{\alpha}\left\langle x, T^{*}\left(\phi_{\alpha} \psi\right)\right\rangle=\lim _{\alpha}\left\langle T(x), \phi_{\alpha} \psi\right\rangle=\langle T(x), \psi\rangle
\end{aligned}
$$

using Propositions 4.1, 5.3 and Theorem 5.6. Since $C^{*}(G)$ is ultraweakly dense in $V N(G)$ and the operators $\hat{m}_{L}$ and $T$ are ultraweakly continuous, it follows that $\hat{m}_{L}=T$.

Corollary 6.7 (McKennon [23]). Assume that $G$ is amenable. Let $T$ be a bounded linear operator from $A(G)$ into $A(G)$. Then the following are equivalent: 
(a) $T(\phi \cdot \gamma)=\phi \cdot T(\gamma)$ for each $\phi, \gamma \in A(G)$.

(b) There exist $m \in B(G)$ such that $T(\gamma)=m \cdot \gamma$ for each $\gamma \in A(G)$ and $\|T\|=\|m\|$.

Consequently the algebra of all multipliers on $A(G)$ (i.e. operators satisfying condition (a)) is isometric and algebra isometric to $B(G)$.

Proof. (b) implies (a) is trivial. Conversely, if $T$ satisfies (a), then $T^{*}$ : $V N(G) \rightarrow V N(G)$ commutes with the action of $A(G)$. By Theorem 6.6, there exists $m \in B(G)$ such that $\hat{m}_{L}=T^{*}$. Then $m$ can easily be verified to satisfy (b).

Remark. (a) Hively [16, Theorem 5.4] proved, using the lifting theorem, that if $G$ is abelian and nondiscrete, then there exists a bounded linear operator $T$ from $L_{\infty}(\hat{G})$ into $L_{\infty}(\hat{G})$ which is not weak ${ }^{*}$ continuous and commutes with translations. Since $B(G)$ is properly contained in $U B C(\hat{G})^{*}$ when $G$ is nondiscrete, Theorem 6.6 implies Hively's result.

(b) Corollary 6.7 is due to Wendel [28] for $G$ abelian.

If $m$ is a topological invariant mean on $U B C(\hat{G})$ (which always exists [25, Theorem 4]) then $m_{L}$ is a compact linear operator commuting with action of $A(G)$ on $V N(G)$. We shall show such operators cannot be ultraweakly continuous unless $G$ is discrete. We first establish the following lemma, which is proved for abelian groups in [20]:

LEMMA 6.8. If $G$ is discrete, then for each $\gamma \in A(G)$, the set $O(\gamma)=\{\phi \cdot \gamma$; $\phi \in A(G)$ and $\|\phi\| \leqslant 1\}$ is relatively compact in the norm topology of $A(G)$. Conversely, if there exist a nonzero $\gamma \in A(G)$ such that $O(\gamma)$ is relatively compact in the weak topology of $A(G)$, then $G$ is discrete.

Proof. If $G$ is discrete, and $\gamma=1_{a}$, then $\phi \cdot 1_{a}=\phi(a) 1_{a}$ for each $\phi \in$ $A(G)$. Hence $O(\gamma) \subseteq\left\{\lambda 1_{a} ;|\lambda| \leqslant 1\right\}$ which is compact. It follows that $O(\gamma)$ is relatively compact in the norm topology for each $\gamma \in\left\langle 1_{a} ; a \in G\right\rangle$. If $\gamma \in$ $A(G)$, then there exists a sequence $\gamma_{n}$ such that $\left\|\gamma_{n}-\gamma\right\| \rightarrow 0$ and each $\gamma_{n}$ has finite support. An argument similar to [14, Theorem 18.3(iv)] shows that $O(\gamma)$ is also relatively compact in the norm topology.

Conversely, if $\gamma$ is a nonzero element in $A(G)$ such that $O(\gamma)$ is relatively compact in the weak topology of $A(G)$, let $K=\overline{O(\gamma)}$. Then $K$ is a convex weakly compact subset of $A(G)$. Let $a \in G$ such that $\gamma(a) \neq 0$ and consider the set $S=\{\phi \in A(G), \phi(a)=1$ and $\|\phi\|=1\}$. Let $\delta=\left\{T_{\phi} ; \phi \in A(G)\right\}$ where $T_{\phi}(\psi)=\phi \cdot \psi$ for each $\psi \in A(G)$. Then $\delta$ is a commuting semigroup of affine continuous mappings from ( $K$, weak) into ( $K$, weak). By the Markov-Kakutani fixed point theorem [6, p. 456], there exists $\psi_{0} \in K$ such that $\phi \cdot \psi_{0}=\psi_{0}$ for each $\phi \in S$. Let $\psi_{1}(t)=\psi\left(a^{-1} t\right)$ for each $t \in G$. Then $\psi_{1} \in A(G), \psi_{1}$ is nonzero, and $\phi \cdot \psi_{1}=\psi_{1}$ for each state $\phi$ in $A(G)$. Replacing $\psi_{1}$ by its real or imaginary part, we may assume that $\psi_{1}$ is selfadjoint. 
Write $\psi_{1}=\psi_{1}^{+}-\psi_{1}^{-}$. Since $\left\|\phi \cdot \psi_{1}^{+}\right\|=\left\|\psi_{1}^{+}\right\|$and $\left\|\phi \cdot \psi_{1}^{-}\right\|=\left\|\psi_{1}^{-}\right\|$for each state $\phi$, it follows that $\phi \cdot \psi_{1}^{+}=\psi_{1}^{+}$and $\phi \cdot \psi_{1}^{-}=\psi_{1}^{-}$for each state $\phi$. Since either $\psi_{1}^{+}$or $\psi_{1}^{-}$is nonzero, it follows from [25, Proposition 5] that $G$ is discrete.

Proposition 6.9. If $G$ is nondiscrete and $T$ is a weakly compact multiplier on $A(G)$, then $T=0$. Consequently, there exist no nonzero operators commuting with action of $A(G)$ on $V N(G)$.

Proposition 6.10. If $G$ is discrete and amenable, then $m \rightarrow \hat{m}_{L}$ is a linear isometry and an algebra isomorphism from $A(G)$ onto the algebra of all ultraweakly continuous (weakly) compact operators commuting with the action of $A(G)$ on $V N(G)$.

Proof. If $m \in A(G)$, and $V(\phi)=m \cdot \phi$ for each $\phi \in A(G)$, then $V^{*}=$ $\hat{m}_{L}$. Since $V$ is compact by Lemma 6.8 , it follows that $\hat{m}_{L}$ is compact. Conversely, if $T$ is a weakly compact ultraweakly continuous operator commuting with the action of $A(G)$ on $V N(G)$, and $\left\{\phi_{\alpha}\right\}$ is a bounded approximate identity on $A(G)$, let $W$ be the restriction of $T^{*}$ to $A(G)$. Then $W$ is also weakly compact. Hence by passsing to a subnet if necessary, we may assume that $W\left(\phi_{\alpha}\right)$ converges to some element $m$ in $A(G)$. Then $T=\hat{m}_{L}$.

Using Proposition 6.10, we can obtain easily:

Proposition 6.11. If $G$ is discrete and amenable, then $A(G)$ is isometric and algebra isomorphic to the algebra of all (weakly) compact multipliers on $A(G)$.

Remark. When $G$ is abelian, Proposition 6.9 is due to Sakai [27, Theorem 1], and Proposition 6.11 is due to Akemann [2], Kitchen [18] and Gaudry [11].

Proposition 6.12. If there exists a nonzero element $m \in A(G)$ such that the map $q_{m}: \psi \rightarrow \psi \cdot m$ from $B(G)$ into $A(G)$ is weak*-weak continuous, then $G$ is discrete. If $G$ is discrete and amenable, then $q_{m}$ is weak*-weak continuous for each $m \in A(G)$.

Proof. The first statement follows from Lemma 6.8. If $G$ is discrete and amenable, then $U B C(\hat{G})=C_{\rho}^{*}(G)=C^{*}(G)$ by Proposition 4.5. Hence if $m \in A(G)$, and $\left\{\phi_{\alpha}\right\}$ is a net in $B(G)$ such that $\left\langle\phi_{\alpha}, x\right\rangle \rightarrow\langle\phi, x\rangle$ for each $x \in C^{*}(G)$, then for each $x \in V N(G),\left\langle\phi_{\alpha} \cdot m, x\right\rangle=\left\langle\phi_{\alpha}, m \cdot x\right\rangle$ converges to $\langle\phi, m \cdot x\rangle$ since $m \cdot x \in C^{*}(G)$.

7. Means on $U B C(\hat{G})$. We assume throughout this section that $G$ is amenable. An application of the Hahn-Banach extension theorem shows that if $G$ is commutative, then the functionals $\left\{\delta_{t} ; t \in \hat{G}\right\}$ where $\delta_{t}(x)=x(t)$ for

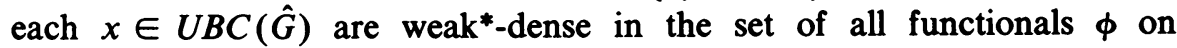


$U B C(\hat{G})$ such that $\|\phi\|=\phi(1)=1$ (see for example [13, p. 1]). In this section, we shall generalise this result to $U B C(\hat{G})$ for amenable $G$ and give some applications.

We let

$$
\begin{aligned}
M & =\left\{\phi \in U B C(\hat{G})^{*} ;\|\phi\|=\phi(1)=1\right\}, \\
P_{1}(G) & =\{\phi \in B(G) ; \phi>0 \text { and }\|\phi\|=1\}, \\
E(G) & =\text { extreme points of } P_{1}(G) .
\end{aligned}
$$

Elements in $M$ are called means.

LEMMA 7.1. (a) If $m \in M$, then $m\left(x^{*}\right)=\overline{m(x)}$ for each $x \in U B C(\hat{G})$. Furthermore, $m(x) \geqslant 0$ if $x \in U B C(G)$ and $x$ is positive (as an element of $V N(G))$.

(b) If $\phi \in P_{1}(G)$, then $\hat{\phi} \in M$.

Proof. (a) Extend $m$ to $V N(G)$ retaining the norm, and use [26, Proposition 1.5.2].

(b) Write $1=\gamma \cdot 1$, where $\gamma \in A(G)$ and $\gamma$ is a state. Then $\langle\hat{\phi}, \gamma \cdot 1\rangle=$ $\langle\phi \gamma, 1\rangle=\phi(e) \gamma(e)=1$.

Leptin [21] proved that for amenable locally compact group $G, A(G)$ has a bounded approximate identity. We need the following slightly stronger assertion.

LEMMA 7.2. $A(G)$ has an approximate identity contained in $P_{1}(G)$.

Proof. Given $\varepsilon>0$, and a compact set $\sigma \subseteq G$, we can find by Reiter's condition [13, p. 44], a function $h \in L_{1}(G)$ such that $h>0,\|h\|_{1}=1$ and $\left\|{ }_{t} h-h\right\|_{1}<\varepsilon$ for all $t \in \sigma$. Define $k=h^{1 / 2}$ and set $\phi_{(e, \sigma)}=k^{*} \tilde{k}$ then $\|\phi(\varepsilon, \sigma)\|=\phi(e)=\|k\|_{2}=1, \phi_{(e, \sigma)} \geqslant 0$, and $\left\{\phi_{(e, \sigma)}\right\}$ converges to 1 in the open-compact topology as shown in [13, p. 61]. Hence by Theorem 3.2, the net is also an approximate identity in $A(G)$.

LEMma 7.3. Given $\phi \in P_{1}(G)$, there exists a net $\left\{\phi_{\alpha}\right\}$ in $\operatorname{co}(E(G) \cup\{0\})[$ or in $A(G) \cap P_{1}(G)$ ] such that $\hat{\phi}_{\alpha}$ converges to $\hat{\phi}$ in the weak ${ }^{*}$-topology of $\operatorname{UBC}(\hat{G})^{*}$.

Proof. Since $\Delta=\{\phi \in \mathrm{P}(\mathrm{G}) ;\|\phi\| \leqslant 1\}$ is weak ${ }^{*}$-compact, we can find a net of convex combinations $\phi_{\alpha}=\sum_{i=1}^{n} \lambda_{i} \gamma_{i}$, such that each $\gamma_{i}$ is an extreme point of $\Delta$ and $\phi_{\alpha}$ converges to $\phi$ in the weak*-topology of $B(G)$. By passing to a subnet, we may assume that $\phi_{\alpha}$ converges to $\phi$ in the $w^{\sim}$-topology. Also each $\gamma_{i}$ is either zero, or has norm 1. Hence $\gamma_{i} \in E(G) \cup\{0\}$.

An application of Thoerem 3.2 shows that $\hat{\phi}_{\alpha}$ converges to $\hat{\phi}$ in the weak*-topology.

To show that a similar net $\left\{\phi_{\alpha}\right\}$ can be chosen from $A(G) \cap P_{1}(G)$, we let 
$\left\{\psi_{\alpha}\right\} \in P_{1}(G) \cap A(G)$ be an approximate identity for $A(G)$ (Lemma 7.2); then $\phi_{\alpha}=\psi_{\alpha} \cdot \phi \in P_{1}(G) \cap A(G)$, and $\hat{\phi}_{\alpha}$ converges to $\hat{\phi}$ in the weak*topology.

THEOREM 7.4. Each of the following sets is weak*-dense in the set of means on $U B C(\hat{G})$ :

(a) $\operatorname{co}\{\hat{\gamma} ; \gamma \in E(G)\}$.

(b) $\left\{\hat{\gamma} ; \gamma \in A(G) \cap P_{1}(G)\right\}$.

(c) $\left\{\hat{\gamma}: \gamma \in P_{1}(G)\right\}$.

Proof. (a) Let $K$ denote the weak*-closure of $\operatorname{co}\{\hat{\gamma} ; \gamma \in E(G)\}$. Clearly $K \subseteq M$. If $m \in M$ and $m \notin K$, by the Hahn-Banach theorem [6, V.2.10], there exist $y \in U B C(\hat{G})$ such that

$$
\operatorname{Re}\langle m, y\rangle>\sup \{\operatorname{Re}\langle\hat{\gamma}, y\rangle ; \hat{\gamma} \in E(G)\} .
$$

Write $x=\left(y+y^{*}\right) / 2$. Then, by Lemma 7.1,

$$
\langle m, x\rangle>\sup \{\langle\hat{\gamma}, x\rangle ; \gamma \in E(G)\}=\alpha
$$

and $x$ is selfadjoint. Write $z=\alpha \cdot 1-\mathrm{x}$. Then $z$ is a positive element in $V N(G)$. In fact, $\langle\hat{\gamma}, z\rangle>0$ for each $\gamma \in E(G)$. If $\gamma \in P_{1}(G) \cap A(G)$, by Lemma 7.2, we can find a net $\gamma_{\alpha} \in \operatorname{co}(E(G) \cup\{0\})$ such that $\hat{\gamma}_{\alpha}$ converges to $\hat{\gamma}$ in the weak*-topology. Hence $\langle\gamma, x\rangle=\langle\hat{\gamma}, x\rangle\rangle 0$. By Lemma 7.1(a), $\langle m, z\rangle>0$, but $\langle m, z\rangle\langle 0$, which is impossible. Hence $m \in K$.

Proof of (b) is similar, and (c) follows from (b).

COROLlary 7.5. If $x$ is a selfadjoint element in $U B C(\hat{G})$, then

$$
\|x\|=\sup \{|\langle\hat{\gamma}, x\rangle| ; \gamma \in E(G)\} .
$$

Proof. By [26, 1.5.4], $\|x\|=\sup \left\{|\langle m, x\rangle| ; m \in V N(G)^{*},\|m\|=m(1)=\right.$ 1). Use Theorem 7.4.

COROLlary 7.6. Let $K$ be a closed convex subset of $A(G)$, then the following are equivalent:

(a) $\phi \cdot K \subseteq K$ for each $\phi \in A(G) \cap P_{1}(G)$.

(b) $\phi \cdot K \subseteq K$ for each $\phi \in P_{1}(G)$.

(c) $\phi \cdot K \subseteq K$ for each $\phi \in E(G)$.

Proof. (a) $\Rightarrow$ (b). Let $\phi \in P_{1}(G)$. By Theorem 7.4, there exists a net $\left\{\phi_{\alpha}\right\}$ in $P_{1}(G) \cap A(G)$ such that $\hat{\phi}_{\alpha} \rightarrow \hat{\phi}$ in the weak ${ }^{*}$-topology of $U B C(\hat{G})^{*}$. Hence if $\gamma \in K$, and $x \in V N(G)$, the net $\left\langle\phi_{\alpha} \cdot \gamma, x\right\rangle=\left\langle\phi_{\alpha}, \gamma \cdot x\right\rangle$ converges to $\langle\phi, \gamma \cdot x\rangle=\langle\phi \cdot \gamma, x\rangle$. Since $\phi_{\alpha} \cdot \gamma \in K$ for each $\alpha$ and $K$ is closed, it follows that $\phi \cdot \gamma \in K$, that (b) $\Rightarrow$ (c) is trivial, and (c) $\Rightarrow(a)$ can be done by an argument similar to that of $(a) \Rightarrow(b)$. 
Corollary 7.7. Let $\psi \in A(G)$. Then

$$
\begin{aligned}
\overline{\operatorname{co}}\{\phi \cdot \psi ; \phi \in E(G)\} & =\overline{\operatorname{co}}\left\{\phi \cdot \psi ; \phi \in P_{1}(G)\right\} \\
& =\overline{\operatorname{co}}\left\{\phi \cdot \psi ; \phi \in P_{1}(G) \cap A(G)\right\} .
\end{aligned}
$$

Proof. Let $K$ be any of the sets and use Corollary 7.6.

Using an argument similar to that for Corollary 7.6, we can also prove:

COROLlaRY 7.8. Let $T$ be a bounded linear operator from $A(G)$ into $A(G)$, the following are equivalent:

(a) $T(\phi \cdot \gamma)=\phi \cdot T(\gamma)$ for each $\phi, \gamma \in A(G)$.

(b) $T(\phi \cdot \gamma)=\phi \cdot T(\gamma)$ for each $\phi \in B(G)$ and $\gamma \in A(G)$.

(c) $T(\phi \cdot \gamma)=\phi \cdot T(\gamma)$ for each $\phi \in E(G)$ and $\gamma \in A(G)$.

REMARK. When $G$ is abelian, elements in $E(G)$ correspond to point evaluations at elements in $\hat{G}$. Hence for $G$ abelian, Corollary 7.6 and Corollary 7.7 are generalisations of our results in [20, Theorem 4.1(a) and Corollary 4.2(a) with $p=1$ ], and Corollary 7.8 is a generalisation of Wendel's result [15, Theorem 35.5]. Also, for the special case that $G$ is abelian and $K$ a closed linear subspace of $A(G)$, Corollary $7.6(a) \Leftrightarrow(c)$ is found in [22, Theorem 31F].

We thank the referee for his many valuable suggestions and for correcting an error in the proof of Proposition 5.3.

\section{REFERENCES} $13,659$.

1. R. Arens, The adjoint of a bilinear operation, Proc. Amer. Math. Soc. 2 (1951), 839-848. MR

2. C. A. Akemann, Some mapping properties of the group algebras of a compact group, Pacific J. Math. 22 (1967), 1-8. MR 35 \# 3458.

3. C. A. Akemann and M. E. Walter, Non-abelian Pontriagin duality, Duke Math. J. 39 (1972), 451-463. MR 47 \#3595.

4. P. C. Curtis, Jr., and A. Figà-Talamanca, Factorization theorems for Banach algebras, Function Algebras (Proc. Internat. Sympos. on Function Algebras, Tulane Univ., 1965), ScottForesman, Chicago, Ill., 1966, pp. 169-185. MR 34 \# 3350.

5. J. Dixmier, Les $C^{*}$-algébres et leurs représentations, Cahiers Scientifiques, Fasc. XXIX, Gauthier-Villars, Paris, 1964. MR 30 \# 1404.

6. N. Dunford and J. T. Schwartz, Linear operators. I, Interscience, New York, 1958. MR 22 \#8302.

7. C. F. Dunkl and D. E. Ramirez, Weakly almost periodic functionals carried by hypercosets, Trans. Amer. Math. Soc. 164 (1972), 427-434. MR 45 \# 463.

8. E. G. Effros, Order ideals in a $C^{*}$-algebra and its dual, Duke Math. J. 30 (1963), 391-411. MR 27 \# 1847.

9. J. A. Ernest, A new group algebra for locally compact groups. I, Amer. J. Math. 86 (1964), 467-492. MR 29 \#4838.

10. P. Eymard, L'algèbre de Fourier d'un groupe localement compact, Bull. Soc. Math. France 92 (1964), 181-236. MR 37 \#4208.

11. G. I. Gaudry, Quasimeasures and multiplier problems, Doctoral dissertation, Australian National Univ., 1966, Canberra, Australia. 
12. E. E. Granirer, Weakly almost periodic and uniformly continuous functionals on the Fourier algebra of any locally compact group, Trans. Amer. Math. Soc. 189 (1974), 371-382. MR 49 \# 1017.

13. F. P. Greenleaf, Invariant means on topological groups and their applications, Van Nostrand Math. Studies, no. 16, Van Nostrand, Reinhold, New York, 1969. MR 40 \#4776.

14. E. Hewitt and K. Ross, Abstract harmonic analysis. I, Springer-Verlag, Berlin; Academic Press, New York, 1963. MR 28 \# 158.

15. __ Abstract harmonic analysis. II, Springer-Verlag, Berlin and New York, 1970. MR 41 \# 7378; erratum, MR 42, p. 1825.

16. G. A. Hively, The representation of norm-continuous multipliers on $L^{\infty}$-spaces, Trans. Amer. Math. Soc. 184 (1973), 343-353. MR 49 \# 11150.

17. J. L. Kelley and Isaac Namioka, Linear topological spaces, Van Nostrand, Princeton, N.J., 1963. MR 29 \# 3851.

18. J. W. Kitchen, Jr., The almost periodic measures on a compact abelian group, Monatsh. Math. 72 (1968), 217-219. MR 37 \#5614.

19. A. T. Lau, Operators which commute with convolutions on subspaces of $L_{\infty}(G)$, Colloq. Math. (to appear).

20. , Closed convex invariant subsets of $L_{p}(G)$, Trans. Amer. Math. Soc. 232 (1977),

131-142.

21. H. Leptin, Sur l'algèbre de Fourier d'un groupe localement compact, C. R. Acad. Sci. Paris Sér. A-B 266 (1968), A1180-A1182. MR 39 \#362.

22. L. H. Loomis, An introduction to abstract harmonic analysis, Van Nostrand, Princeton, N. J., 1953. MR 14, 883.

23. Kelly McKennon, Multipliers, positive functionals, pasitive-definite functions and FourierStieltjes transforms, Mem. Amer. Math. Soc. no. 111 (1971).

24. D. A. Raukov, On various types of convergence of positive definite functions, Dokl. Akad. Nauk SSSR (N.S.) 58 (1947), 1279-1282. (Russian) MR 9, 328.

25. P. F. Renaud, Invariant means on a class of von Neumann algebras, Trans. Amer. Math. Soc. 170 (1972), 285-291.

26. S. Sakai, $C^{*}$-algebras and $W^{*}$-algebras, Springer-Verlag, Berlin and New York, 1971.

27. $\longrightarrow$ Weakly compact operators on operator algebras, Pacific J. Math. 14 (1964), $659-664$. MR 29 \# 488.

28. J. G. Wendel, Left centralizers and isomorphisms of group algebras, Pacific J. Math. 2 (1952), 251-261. MR 14, 246.

29. James C. S. Wong, Topologically stationary locally compact groups and amenability, Trans. Amer. Math. Soc. 144 (1969), 351-363. MR 40 \#2781.

Department of Mathematics, University of Alberta, Edmonton, Alberta, Canada T6G 2G1 